\title{
Logarithmic Bounds for Oscillatory Singular Integrals on Hardy Spaces
}

\author{
Hussain Al-Qassem, ${ }^{1}$ Leslie Cheng, ${ }^{2}$ and Yibiao Pan $^{3}$ \\ ${ }^{1}$ Department of Mathematics and Physics, Qatar University, Doha, Qatar \\ ${ }^{2}$ Department of Mathematics, Bryn Mawr College, Bryn Mawr, PA 19010, USA \\ ${ }^{3}$ Department of Mathematics, University of Pittsburgh, Pittsburgh, PA 15260, USA
}

Correspondence should be addressed to Yibiao Pan; yibiao@pitt.edu

Received 28 December 2015; Accepted 7 February 2016

Academic Editor: Maria Alessandra Ragusa

Copyright (C) 2016 Hussain Al-Qassem et al. This is an open access article distributed under the Creative Commons Attribution License, which permits unrestricted use, distribution, and reproduction in any medium, provided the original work is properly cited.

We establish a logarithmic bound for oscillatory singular integrals with quadratic phases on the Hardy space $H^{1}\left(\mathbb{R}^{n}\right)$. The logarithmic rate of growth is the best possible.

\section{Introduction}

For $n \in \mathbb{N}$, let $K(x)$ be a Calderón-Zygmund kernel on $\mathbb{R}^{n}$ and let $P(x)$ be a polynomial of $n$ variables with real coefficients. Consider the following oscillatory singular integral operator:

$$
T_{P}: f \longrightarrow \text { p.v. } \int_{\mathbb{R}^{n}} e^{i P(x-y)} K(x-y) f(y) d y \text {. }
$$

It is well known that $T_{P}$ is bounded from $L^{p}\left(\mathbb{R}^{n}\right)$ to $L^{p}\left(\mathbb{R}^{n}\right)$ when $1<p<\infty$ and also from $L^{1}\left(\mathbb{R}^{n}\right)$ to $L^{1, \infty}\left(\mathbb{R}^{n}\right)$. Additionally, $L^{p} \rightarrow L^{p}$ and $L^{1} \rightarrow L^{1, \infty}$ bounds are dependent on the degree of the phase polynomial $P$ only, not its coefficients $($ see $[1,2])$.

However, for $H^{1}\left(\mathbb{R}^{n}\right) \rightarrow H^{1}\left(\mathbb{R}^{n}\right)$ boundedness of $T_{P}$, the answers are not nearly as clear-cut. First, it was shown in [3] that, in general, $T_{P}$ may fail to be bounded on $H^{1}\left(\mathbb{R}^{n}\right)$ and when the coefficients of the first-order terms of $P$ vanish, $T_{P}$ is bounded from $H^{1}\left(\mathbb{R}^{n}\right)$ to itself with a bound independent of the higher order coefficients of $P$.

More recent work can be found in $[4,5]$, including the following.

Theorem 1 (see [5]). Let $n \in \mathbb{N}, m \geq 2$, and $P(x)=$ $\sum_{0 \leq|\alpha| \leq m} a_{\alpha} x^{\alpha}$ be a polynomial of degree $m$ in $\mathbb{R}^{n}$ with real coefficients. Let $K$ be a Calderón-Zygmund kernel and let $T_{P}$ be given as in (1). Then, there exists a positive constant $C$ such that

$$
\left\|T_{P} f\right\|_{H^{1}\left(\mathbb{R}^{n}\right)} \leq C\left(1+\frac{\sum_{|\alpha|=1}\left|a_{\alpha}\right|}{\sum_{2 \leq|\alpha| \leq m}\left|a_{\alpha}\right|^{1 /|\alpha|}}\right)\|f\|_{H^{1}\left(\mathbb{R}^{n}\right)}
$$

for all $f \in H^{1}\left(\mathbb{R}^{n}\right)$. The constant $C$ may depend on $n, m$, and $K$ but is independent of the coefficients $\left\{a_{\alpha}\right\}$ of $P$.

In order to determine the optimal bound on $\left\|T_{P}\right\|_{H^{1} \rightarrow H^{1}}$, an example was given in [5] to show that, as $\sum_{|\alpha|=1}\left|a_{\alpha}\right| / \sum_{2 \leq|\alpha| \leq m}\left|a_{\alpha}\right|^{1 /|\alpha|} \rightarrow \infty$, any bound on $\left\|T_{P}\right\|_{H^{1} \rightarrow H^{1}}$ must increase at least at the rate of $\log \left(\sum_{|\alpha|=1}\left|a_{\alpha}\right| / \sum_{2 \leq|\alpha| \leq m}\left|a_{\alpha}\right|^{1 /|\alpha|}\right)$. This naturally leads to the following question.

Does

$$
\begin{aligned}
& \left\|T_{P} f\right\|_{H^{1}\left(\mathbb{R}^{n}\right)} \\
& \quad \leq C_{n, m}\left(1+\log ^{+}\left(\frac{\sum_{|\alpha|=1}\left|a_{\alpha}\right|}{\sum_{2 \leq|\alpha| \leq m}\left|a_{\alpha}\right|^{1 /|\alpha|}}\right)\right)\|f\|_{H^{1}\left(\mathbb{R}^{n}\right)}
\end{aligned}
$$

hold for all $f \in H^{1}\left(\mathbb{R}^{n}\right)$ ?

In this paper, we will prove that the answer to the above question is affirmative for all quadratic polynomials. Namely, we have the following. 
Theorem 2. Let $n \in \mathbb{N}$ and $P(x)=\sum_{0 \leq|\alpha| \leq 2} a_{\alpha} x^{\alpha}$ be $a$ quadratic polynomial in $\mathbb{R}^{n}$ with real coefficients. Let $K$ be a Calderón-Zygmund kernel and let $T_{P}$ be given as in (1). Then, there exists a positive constant $C$ such that

$$
\begin{aligned}
& \left\|T_{P} f\right\|_{H^{1}\left(\mathbb{R}^{n}\right)} \\
& \quad \leq C\left(1+\log ^{+}\left(\frac{\sum_{|\alpha|=1}\left|a_{\alpha}\right|}{\sum_{|\alpha|=2}\left|a_{\alpha}\right|^{1 / 2}}\right)\right)\|f\|_{H^{1}\left(\mathbb{R}^{n}\right)}
\end{aligned}
$$

for all $f \in H^{1}\left(\mathbb{R}^{n}\right)$. The constant $C$ may depend on $n$ and $K$ but is independent of the coefficients $\left\{a_{\alpha}\right\}$ of $P$.

We point out that $C$ denotes an absolute constant whose value may change from line to line.

\section{Some Definitions and Lemmas}

Many of the tools we use are known. For readers who wish to see the definitions and some of their properties, the following references are suggested: [6-12].

For $x \in \mathbb{R}^{n}$ and $r>0$, let $B(x, r)=\left\{y \in \mathbb{R}^{n}:|y-x|<r\right\}$ and $|B(x, r)|$ denote the Euclidean volume of $B(x, r)$.

Let $\phi$ be a function in the Schwartz space $\mathcal{S}\left(\mathbb{R}^{n}\right)$ such that $\int_{\mathbb{R}^{n}} \phi(x) d x=1$. For each $f \in L_{\text {loc }}^{1}\left(\mathbb{R}^{n}\right)$ and $x \in \mathbb{R}^{n}$, we let

$$
M_{\phi} f(x)=\sup _{s>0}\left|\left(f * \phi_{s}\right)(x)\right|
$$

where $\phi_{s}(x)=s^{-n} \phi(x / s)$.

Definition 3. For a nonnegative, locally integrable function $w$ on $\mathbb{R}^{n}$, the Hardy space $H^{1}\left(\mathbb{R}^{n}\right)$ is given by

$$
H^{1}\left(\mathbb{R}^{n}\right)=\left\{f \in L_{\mathrm{loc}}^{1}\left(\mathbb{R}^{n}\right):\left\|M_{\phi} f\right\|_{L^{1}\left(\mathbb{R}^{n}\right)}<\infty\right\},
$$

with $\|f\|_{H^{1}\left(\mathbb{R}^{n}\right)}=\left\|M_{\phi} f\right\|_{L^{1}\left(\mathbb{R}^{n}\right)}$.

Definition 4. A measurable function $f$ on $\mathbb{R}^{n}$ is called $H^{1}$ atom if there exist $\zeta \in \mathbb{R}^{n}$ and $r>0$ such that

$$
\begin{aligned}
\operatorname{supp}(f) & \subseteq B(\zeta, r) ; \\
\|f\|_{\infty} & \leq \frac{1}{|B(\zeta, r)|} ; \\
\int_{\mathbb{R}^{n}} f(y) d y & =0 .
\end{aligned}
$$

Lemma 5 (see $[9,10])$. For each $f \in H^{1}\left(\mathbb{R}^{n}\right)$, there exist $H^{1}$ atoms $\left\{f_{\gamma}\right\}$ and coefficients $\left\{\omega_{\gamma}\right\}$ such that

$$
\begin{aligned}
f & =\sum_{\nu} \omega_{\nu} f_{\nu}, \\
\|f\|_{H^{1}\left(\mathbb{R}^{n}\right)} & \approx \inf \sum_{\nu}\left|\omega_{\nu}\right| .
\end{aligned}
$$

Definition 6. A $C^{1}$ function $K: \mathbb{R}^{n} \backslash\{0\} \rightarrow \mathbb{C}$ is called a Calderón-Zygmund kernel if the following are true:

(i) There exists $C>0$ such that

$$
|K(x)|+|x||\nabla K(x)| \leq A|x|^{-n}
$$

holds for all $x \in \mathbb{R}^{n} \backslash\{0\}$.

(ii) For all $0<a<b$,

$$
\int_{B(0, b) \backslash B(0, a)} K(x) d x=0 .
$$

Lemma 7. Let $P(x)=\sum_{0 \leq|\alpha| \leq 2} a_{\alpha} x^{\alpha}$ for $x \in \mathbb{R}^{n}$ and $\lambda \geq 0$. Define operator $U_{P, \lambda}$ by

$$
\left(U_{P, \lambda} f\right)(x)=\frac{\chi_{B(0, \lambda)^{c}}(x)}{|x|^{n}} \int_{B(0,1)} e^{i P(x-y)} f(y) d y .
$$

Then, there exists $C>0$ independent of $P$ such that

$$
\left\|U_{P, \lambda} f\right\|_{L^{1}\left(\mathbb{R}^{n}\right)} \leq C\|f\|_{L^{\infty}(B(0,1))}
$$

holds for all $f \in L^{\infty}(B(0,1))$ and $\lambda \geq\left(\sum_{|\alpha|=2}\left|a_{\alpha}\right|^{1 / 2}\right)^{-2}$.

Proof. We start by treating the more difficult case $n \geq 2$. The other case, $n=1$, will be briefly considered later.

Write

$$
\sum_{|\alpha|=2} a_{\alpha} x^{\alpha}=\sum_{j=1}^{n} \sum_{k=1}^{n} b_{j k} x_{j} x_{k}
$$

with $b_{j k}=b_{k j}$ for $1 \leq j, k \leq n$. Then, there exist $l, s \in$ $\{1, \ldots, n\}$ such that

$$
\left|b_{l s}\right|=\max \left\{\left|b_{j k}\right|: 1 \leq j, k \leq n\right\} .
$$

Thus, we have

$$
2 n^{4}\left|b_{l s}\right| \lambda \geq \lambda\left(\sum_{|\alpha|=2}\left|a_{\alpha}\right|^{1 / 2}\right)^{2}>1 .
$$

For $x, y \in \mathbb{R}^{n}$, let

$$
\begin{aligned}
x^{\prime} & =\left(x_{1}, \ldots, x_{l-1}, x_{l+1}, \ldots, x_{n}\right), \\
\tilde{y} & =\left(y_{1}, \ldots, y_{s-1}, y_{s+1}, \ldots, y_{n}\right) .
\end{aligned}
$$

Then, there are polynomials $Q_{1}(\cdot), Q_{2}(\cdot)$ on $\mathbb{R}^{n}, Q_{3}(\cdot), Q_{4}(\cdot)$ on $\mathbb{R}^{n-1}$, and $Q_{5}(\cdot)$ on $\mathbb{R}^{n-1} \times \mathbb{R}^{n-1}$ such that

$$
\begin{aligned}
\sum_{|\alpha|=2} a_{\alpha}(x-y)^{\alpha}= & -2 b_{l s} x_{l} y_{s}+Q_{1}(x)+Q_{2}(y) \\
& +x_{l} Q_{3}(\tilde{y})+y_{s} Q_{4}\left(x^{\prime}\right) \\
& +Q_{5}\left(x^{\prime}, \tilde{y}\right) .
\end{aligned}
$$


Journal of Function Spaces

3

Let $g(x)=f(x)$ for $x \in B(0,1)$ and $g(x)=0$ if $x \in B(0,1)^{c}$.

Then,

$$
\begin{aligned}
& \left\|U_{P, \lambda} f\right\|_{L^{1}\left(\mathbb{R}^{n}\right)}=\int_{\mathbb{R}^{n} \backslash B(0, \lambda)}\left|\int_{\mathbb{R}^{n}} e^{i P(x-y)} g(y) d y\right| \frac{d x}{|x|^{n}} \\
& \quad=\int_{\mathbb{R}^{n} \backslash B(0, \lambda)}\left|\int_{\tilde{y} \in \mathbb{R}^{n-1}} e^{i\left(P(0)+\sum_{|\alpha|=1} a_{\alpha} x^{\alpha}+Q_{1}(x)+x_{l} Q_{3}(\tilde{y})+Q_{5}\left(x^{\prime}, \tilde{y}\right)\right)}\left(\int_{y_{s} \in \mathbb{R}} e^{i\left(-2 b_{l s} x_{l} y_{s}-\sum_{|\alpha|=1} a_{\alpha} y^{\alpha}+Q_{2}(y)+y_{s} Q_{4}\left(x^{\prime}\right)\right)} g(y) d y_{s}\right) d \tilde{y}\right| \frac{d x}{|x|^{n}} \\
& \quad \leq C \int_{x^{\prime} \in \mathbb{R}^{n-1}} \int_{\tilde{y} \in \mathbb{R}^{n-1}} \int_{x_{l} \in \mathbb{R}} h_{x^{\prime}}\left(x_{l}\right)\left|\int_{y_{s} \in \mathbb{R}} e^{-i\left(2 b_{l s} x_{l}\right) y_{s}} g_{x^{\prime}, \tilde{y}}\left(y_{s}\right) d y_{s}\right| d x_{l} d \widetilde{y} d x^{\prime},
\end{aligned}
$$

where

$$
\begin{aligned}
g_{x^{\prime}, \tilde{y}}\left(y_{s}\right) & =e^{i\left(-\sum_{|\alpha|=1} a_{\alpha} y^{\alpha}+\mathrm{Q}_{2}(y)+y_{s} \mathrm{Q}_{4}\left(x^{\prime}\right)\right)} g(y), \\
h_{x^{\prime}}\left(x_{l}\right) & =\frac{\chi_{\left[\lambda^{2}, \infty\right)}\left(\left|x_{l}\right|^{2}+\left|x^{\prime}\right|^{2}\right)}{\left(\left|x_{l}\right|^{2}+\left|x^{\prime}\right|^{2}\right)^{n / 2}} .
\end{aligned}
$$

Since $\left|g_{x^{\prime}, \tilde{y}}\left(y_{s}\right)\right|=|g(y)|$ and $\operatorname{supp}\left(g_{x^{\prime}, \tilde{y}}\right) \subseteq[-1,1]$, we have

$$
\begin{aligned}
& \left\|U_{P, \lambda} f\right\|_{L^{1}\left(\mathbb{R}^{n}\right)} \leq C \int_{x^{\prime} \in \mathbb{R}^{n-1}} \int_{\tilde{y} \in \mathbb{R}^{n-1}} \int_{x_{l} \in \mathbb{R}} h_{x^{\prime}}\left(x_{l}\right) \\
& \cdot\left|\widehat{g_{x^{\prime}, y}}\left(2 b_{l s} x_{l}\right)\right| d x_{l} d \tilde{y} d x^{\prime} \\
& \leq C \int_{x^{\prime} \in \mathbb{R}^{n-1}} \int_{\tilde{y} \in \mathbb{R}^{n-1}}\left(\int_{\mathbb{R}}\left|h_{x^{\prime}}\left(x_{l}\right)\right|^{2} d x_{l}\right)^{1 / 2} \\
& \cdot\left(\int_{\mathbb{R}}\left|\widehat{g_{x^{\prime}, y}}\left(2 b_{l s} x_{l}\right)\right|^{2} d x_{l}\right)^{1 / 2} d \tilde{y} d x^{\prime} \\
& =C\left|b_{l s}\right|^{-1 / 2} \int_{x^{\prime} \in \mathbb{R}^{n-1}} \int_{\tilde{y} \in \mathbb{R}^{n-1}}\left(\int_{\mathbb{R}}\left|h_{x^{\prime}}\left(x_{l}\right)\right|^{2} d x_{l}\right)^{1 / 2} \\
& \cdot\left(\int_{\mathbb{R}}\left|g_{x^{\prime}, \tilde{y}}\left(y_{s}\right)\right|^{2} d y_{s}\right)^{1 / 2} d \widetilde{y} d x^{\prime} \\
& \leq C\left|b_{l s}\right|^{-1 / 2}\|f\|_{L^{\infty}(B(0,1))} \\
& \cdot\left(\int_{\left|x^{\prime}\right| \geq \lambda}\left(\int_{\mathbb{R}} \frac{d x_{l}}{\left(\left|x_{l}\right|^{2}+\left|x^{\prime}\right|^{2}\right)^{n}}\right)^{1 / 2} d x^{\prime}\right. \\
& \left.+\int_{\left|x^{\prime}\right|<\lambda}\left(\int_{\left|x_{l}\right| \geq \sqrt{\lambda^{2}-\left|x^{\prime}\right|^{2}}} \frac{d x_{l}}{\left(\left|x_{l}\right|^{2}+\left|x^{\prime}\right|^{2}\right)^{n}}\right)^{1 / 2} d x^{\prime}\right) \\
& \leq C\left|b_{l s}\right|^{-1 / 2}\|f\|_{L^{\infty}(B(0,1))}\left[\int_{\left|x^{\prime}\right| \geq \lambda} \frac{d x^{\prime}}{\left|x^{\prime}\right|^{n-1 / 2}}\right. \\
& \left.+\lambda^{(1-2 n) / 2} \int_{\left|x^{\prime}\right|<\lambda}\left(\int_{1}^{\infty} \frac{d t}{t^{n} \sqrt{t-\left|x^{\prime} / \lambda\right|^{2}}}\right)^{1 / 2} d x^{\prime}\right]
\end{aligned}
$$

$$
\begin{aligned}
& \leq C\left|b_{l s}\right|^{-1 / 2}\|f\|_{L^{\infty}(B(0,1))}\left(\lambda^{-1 / 2}+\lambda^{-n+1 / 2} \int_{\left|x^{\prime}\right|<\lambda}(1\right. \\
& \left.\left.-\left|\frac{x^{\prime}}{\lambda}\right|^{2}\right)^{-1 / 4} d x^{\prime}\right) \leq C\left(\lambda\left|b_{l s}\right|\right)^{-1 / 2} \\
& \cdot\|f\|_{L^{\infty}(B(0,1))} \leq C\|f\|_{L^{\infty}(B(0,1))} .
\end{aligned}
$$

The treatment of the case $n=1$ only involves the Fourier transform step of the preceding argument. Details are omitted.

Lemma 8. Let $n \in \mathbb{N}$ and $P(x)=\sum_{0 \leq|\alpha| \leq 2} a_{\alpha} x^{\alpha}$ be a quadratic polynomial in $\mathbb{R}^{n}$ with real coefficients. Let $K$ be a CalderonZygmund kernel satisfying (11)-(12) and let $T_{P}$ be given as in (1). Then, there exists a positive constant $C$ such that

$$
\left\|T_{P} f\right\|_{L^{1}\left(\mathbb{R}^{n}\right)} \leq C\left(1+\log ^{+}\left(\frac{\sum_{|\alpha|=1}\left|a_{\alpha}\right|}{\sum_{|\alpha|=2}\left|a_{\alpha}\right|^{1 / 2}}\right)\right)
$$

for every $H^{1}$ atom $f$ which satisfies (7)-(9) with $\zeta=0$ and $r=$ 1. The constant $C$ may depend on $n$ and $A$ but is independent of $\left\{a_{\alpha}\right\}, K$, and $f$.

Proof. By the uniform boundedness of $T_{P}$ on $L^{2}\left(\mathbb{R}^{n}\right)$ and (7)(8),

$$
\begin{aligned}
\int_{B(0,2)}\left|T_{P} f(x)\right| d x & \leq|B(0,2)|^{1 / 2}\left\|T_{P} f\right\|_{L^{2}\left(\mathbb{R}^{n}\right)} \\
& \leq C\|f\|_{L^{2}\left(\mathbb{R}^{n}\right)} \leq C .
\end{aligned}
$$

By (11), we have

$$
\begin{aligned}
& \int_{\mathbb{R}^{n} \backslash B(0,2)}\left|T_{P} f(x)-K(x) \int_{B(0,1)} e^{i P(x-y)} f(y) d y\right| d x \\
& \quad \leq \int_{\mathbb{R}^{n} \backslash B(0,2)} \int_{B(0,1)}|K(x-y)-K(x)||f(y)| d y d x \\
& \quad \leq C\|f\|_{L^{1}\left(\mathbb{R}^{n}\right)} \int_{\mathbb{R}^{n} \backslash B(0,2)}|x|^{-n-1} d x \leq C .
\end{aligned}
$$


Let $\lambda=\left(\sum_{|\alpha|=2}\left|a_{\alpha}\right|^{1 / 2}\right)^{-2}$. It follows from (11) and (7)-(8) and Lemma 7 that

$$
\begin{aligned}
& \int_{\mathbb{R}^{n} \backslash B(0, \max \{2, \lambda\})}\left|T_{P} f(x)\right| d x \\
& \quad \leq C+\int_{\mathbb{R}^{n} \backslash B(0, \lambda)}|K(x)|\left|\int_{B(0,1)} e^{i P(x-y)} f(y) d y\right| d x \\
& \quad \leq C+C\left\|U_{P, \lambda} f\right\|_{L^{1}\left(\mathbb{R}^{n}\right)} \leq C .
\end{aligned}
$$

If $\lambda \leq 2$, then (23) follows from (24) and (26).

Thus, we may assume that $\lambda>2$. To finish the proof, it suffices to show that

$$
\begin{aligned}
& \int_{B(0, \lambda) \backslash B(0,2)}\left|T_{P} f(x)\right| d x \\
& \quad \leq C\left(1+\log ^{+}\left(\frac{\sum_{|\alpha|=1}\left|a_{\alpha}\right|}{\sum_{|\alpha|=2}\left|a_{\alpha}\right|^{1 / 2}}\right)\right) .
\end{aligned}
$$

We will establish (27) by discussing two cases.

Case $1\left(\sum_{|\alpha|=1}\left|a_{\alpha}\right| \geq 1 / 2\right)$. In this case, we have

$$
\begin{aligned}
& \int_{B(0, \lambda) \backslash B(0,2)}\left|T_{P} f(x)\right| d x \\
& \quad \leq C \int_{B(0, \lambda) \backslash B(0,2)} \int_{B(0,1)}|x-y|^{-n}|f(y)| d y d x \\
& \quad \leq C\|f\|_{L^{1}\left(\mathbb{R}^{n}\right)} \int_{B(0, \lambda) \backslash B(0,2)}|x|^{-n} d x \\
& \quad \leq C \ln \left(\frac{1}{2\left(\sum_{|\alpha|=2}\left|a_{\alpha}\right|^{1 / 2}\right)^{2}}\right) \\
& \quad \leq C\left(\ln 2+2 \ln \left(\frac{\sum_{|\alpha|=1}\left|a_{\alpha}\right|}{\sum_{|\alpha|=2}\left|a_{\alpha}\right|^{1 / 2}}\right)\right) \\
& \quad \leq C\left(1+\log ^{+}\left(\frac{\sum_{|\alpha|=1}\left|a_{\alpha}\right|}{\sum_{|\alpha|=2}\left|a_{\alpha}\right|^{1 / 2}}\right)\right) .
\end{aligned}
$$

Case $2\left(\sum_{|\alpha|=1}\left|a_{\alpha}\right|<1 / 2\right)$. In this case, we let

$$
Q(x)=P(0)+\sum_{|\alpha|=2} a_{\alpha} x^{\alpha} .
$$

It follows from Theorem 1 of [3] that

$$
\left\|T_{\mathrm{Q}} f\right\|_{L^{1}\left(\mathbb{R}^{n}\right)} \leq C .
$$

For $x \in \mathbb{R}^{n}$ and $y \in B(0,1)$, we have

$$
\left|e^{i P(x-y)}-e^{i\left(\sum_{|\alpha|=1} a_{\alpha} x^{\alpha}+Q(x-y)\right)}\right| \leq \sum_{|\alpha|=1}\left|a_{\alpha}\right| .
$$

By (30)-(31) and

$$
\sup _{0<t<1 / 2} t \ln \left(\frac{1}{t}\right)=\frac{1}{e}
$$

we have

$$
\begin{aligned}
& \int_{B(0, \lambda) \backslash B(0,2)}\left|T_{P} f(x)\right| d x \leq\left\|T_{Q} f\right\|_{L^{1}\left(\mathbb{R}^{n}\right)} \\
& +\int_{B(0, \lambda) \backslash B(0,2)}\left|T_{P} f(x)-e^{i\left(\sum_{|\alpha|=1} a_{\alpha} \alpha^{\alpha}\right)} T_{Q} f(x)\right| d x \\
& \leq C+C\left(\sum_{|\alpha|=1}\left|a_{\alpha}\right|\right)\|f\|_{L^{1}\left(\mathbb{R}^{n}\right)} \int_{B(0, \lambda) \backslash B(0,2)}|x|^{-n} d x \\
& \quad \leq C+C\left(\sum_{|\alpha|=1}\left|a_{\alpha}\right|\right) \ln \left(\frac{1}{\sum_{|\alpha|=2}\left|a_{\alpha}\right|^{1 / 2}}\right)=C \\
& +C\left(\sum_{|\alpha|=1}\left|a_{\alpha}\right|\right) \\
& \quad\left[\ln \left(\frac{1}{\sum_{|\alpha|=1}\left|a_{\alpha}\right|}\right)+\ln \left(\frac{\sum_{|\alpha|=1}\left|a_{\alpha}\right|}{\sum_{|\alpha|=2}\left|a_{\alpha}\right|^{1 / 2}}\right)\right] \\
& \quad \leq C\left(1+\log ^{+}\left(\frac{\sum_{|\alpha|=1}\left|a_{\alpha}\right|}{\sum_{|\alpha|=2}\left|a_{\alpha}\right|^{1 / 2}}\right)\right) .
\end{aligned}
$$

Thus, (27) holds in both cases.

\section{Proof of Main Theorem}

To finish the proof, we recall the following result concerning Riesz transforms and Hardy spaces.

Lemma 9 (see $[10,13]$ ). For $1 \leq j \leq n$, let $R_{j}$ denote the $j$ th Riesz transform; that is,

$$
\widehat{R_{j} f}(\xi)=\frac{i \xi_{j}}{|\xi|} \widehat{f}(\xi)
$$

Then, there exist $C, C_{1}, C_{2}>0$ such that

$$
\left\|R_{j} f\right\|_{H^{1}\left(\mathbb{R}^{n}\right)} \leq C\|f\|_{H^{1}\left(\mathbb{R}^{n}\right)}
$$

for $1 \leq j \leq n$, and

$$
\begin{aligned}
C_{1}\|f\|_{H^{1}\left(\mathbb{R}^{n}\right)} & \leq\|f\|_{L^{1}\left(\mathbb{R}^{n}\right)}+\sum_{j=1}^{n}\left\|R_{j} f\right\|_{L^{1}\left(\mathbb{R}^{n}\right)} \\
& \leq C_{2}\|f\|_{H^{1}\left(\mathbb{R}^{n}\right)}
\end{aligned}
$$

for all $f \in H^{1}\left(\mathbb{R}^{n}\right)$.

We will now give the proof of Theorem 2.

Proof. For $f \in H^{1}\left(\mathbb{R}^{n}\right)$, let $\left\{\omega_{\gamma}\right\}$ be a sequence of complex numbers and let $\left\{f_{\nu}\right\}$ be a sequence of $H^{1}$ atoms such that

$$
f=\sum_{\nu} \omega_{\nu} f_{\nu}
$$


For each $v$, let $\zeta_{v} \in \mathbb{R}^{n}$ and $r_{v}>0$ such that $\operatorname{supp}\left(f_{v}\right) \subseteq$ $B\left(\zeta_{\nu}, r_{\nu}\right)$ and $\left\|f_{\nu}\right\|_{\infty} \leq\left|B\left(\zeta_{\nu}, r_{\nu}\right)\right|^{-1}=|B(0,1)|^{-1} r_{\nu}^{-n}$. Then,

$$
\begin{aligned}
& r_{\nu}^{n} T_{P} f_{\nu}\left(r_{\nu} x+\zeta_{\nu}\right) \\
& \quad=\text { p.v. } \int_{\mathbb{R}^{n}} e^{i P_{\nu}(x-y)} K_{\nu}(x-y)\left(r_{\nu}^{n} f_{\nu}\left(r_{\nu} y+\zeta_{\nu}\right)\right) d y
\end{aligned}
$$

where $P_{\nu}(x)=P\left(r_{\gamma} x\right)$ and $K_{\nu}(x)=r_{\nu}^{n} K\left(r_{\gamma} x\right)$. Observe that, for each $v, K_{v}$ satisfies (11)-(12) with the same constant $A$ and $r_{\nu}^{n} f_{\nu}\left(r_{\nu} y+\zeta_{\nu}\right)$ satisfies (7)-(9) with $\zeta=0, r=1$. Since

$$
P_{v}(x)=\sum_{0 \leq|\alpha| \leq 2} r_{v}^{|\alpha|} a_{\alpha} x^{\alpha}
$$

by Lemma 8 ,

$$
\begin{aligned}
\left\|T_{P} f_{\nu}\right\|_{L^{1}\left(\mathbb{R}^{n}\right)} & =\int_{\mathbb{R}^{n}}\left|r_{\nu}^{n} T_{P} f_{\nu}\left(r_{\nu} x+\zeta_{v}\right)\right| d x \\
& =C\left(1+\log ^{+}\left(\frac{\sum_{|\alpha|=1}\left|a_{\alpha}\right|}{\sum_{|\alpha|=2}\left|a_{\alpha}\right|^{1 / 2}}\right)\right),
\end{aligned}
$$

which implies that

$$
\begin{aligned}
& \left\|T_{P} f\right\|_{L^{1}\left(\mathbb{R}^{n}\right)} \\
& \quad \leq C\left(1+\log ^{+}\left(\frac{\sum_{|\alpha|=1}\left|a_{\alpha}\right|}{\sum_{|\alpha|=2}\left|a_{\alpha}\right|^{1 / 2}}\right)\right)\left(\sum_{\nu}\left|\omega_{\nu}\right|\right) .
\end{aligned}
$$

It follows from Lemma 5 that

$$
\begin{aligned}
& \left\|T_{P} f\right\|_{L^{1}\left(\mathbb{R}^{n}\right)} \\
& \quad \leq C\left(1+\log ^{+}\left(\frac{\sum_{|\alpha|=1}\left|a_{\alpha}\right|}{\sum_{|\alpha|=2}\left|a_{\alpha}\right|^{1 / 2}}\right)\right)\|f\|_{H^{1}\left(\mathbb{R}^{n}\right)} .
\end{aligned}
$$

By the translation invariance of $T_{P}$ and (42) and (35), we have

$$
\begin{aligned}
\sum_{j=1}^{n} & \left\|R_{j} T_{P} f\right\|_{L^{1}\left(\mathbb{R}^{n}\right)}=\sum_{j=1}^{n}\left\|T_{P} R_{j} f\right\|_{L^{1}\left(\mathbb{R}^{n}\right)} \\
& \leq C\left(1+\log ^{+}\left(\frac{\sum_{|\alpha|=1}\left|a_{\alpha}\right|}{\sum_{|\alpha|=2}\left|a_{\alpha}\right|^{1 / 2}}\right)\right) \\
& \cdot\left(\sum_{j=1}^{n}\left\|R_{j} f\right\|_{H^{1}\left(\mathbb{R}^{n}\right)}\right) \\
& \leq C\left(1+\log ^{+}\left(\frac{\sum_{|\alpha|=1}\left|a_{\alpha}\right|}{\sum_{|\alpha|=2}\left|a_{\alpha}\right|^{1 / 2}}\right)\right)\|f\|_{H^{1}\left(\mathbb{R}^{n}\right)} .
\end{aligned}
$$

By applying (36), (42), and (43), we obtain (4).

\section{Conflict of Interests}

The authors declare that there is no conflict of interests regarding the publication of this paper.

\section{References}

[1] F. Ricci and E. M. Stein, "Harmonic analysis on nilpotent groups and singular integrals. I. Oscillatory integrals," Journal of Functional Analysis, vol. 73, no. 1, pp. 179-194, 1987.

[2] S. Chanillo and M. Christ, "Weak $(1,1)$ bounds for oscillatory singular integrals," Duke Mathematical Journal, vol. 55, no. 1, pp. 141-155, 1987.

[3] Y. Hu and Y. Pan, "Boundedness of oscillatory singular integrals on Hardy spaces," Arkiv för Matematik, vol. 30, no. 2, pp. 311320, 1992.

[4] M. Folch-Gabayet and J. Wright, "Weak-type (1,1) bounds for oscillatory singular integrals with rational phases," Studia Mathematica, vol. 210, no. 1, pp. 57-76, 2012.

[5] H. Al-Qassem, L. Cheng, and Y. Pan, "Estimates for oscillatory singular integrals on Hardy spaces," Studia Mathematica, vol. 224, no. 3, pp. 277-289, 2014.

[6] M. Bramanti and M. C. Cerutti, " $W_{p}^{1,2}$ solvability for the Cauchy-Dirichlet problem for parabolic equations with VMO coefficients," Communications in Partial Differential Equations, vol. 18, no. 9-10, pp. 1735-1763, 1993.

[7] F. Chiarenza, M. Frasca, and P. Longo, "Interior $\mathbf{W}^{2, \mathbf{p}}$ estimates for nondivergence elliptic equations with discontinuous coefficients," Ricerche di Matematica, vol. 40, no. 1, pp. 149-168, 1991.

[8] F. Chiarenza, M. Frasca, and P. Longo, " $\mathrm{W}^{2, \mathrm{p}}$-solvability of the Dirichlet problem for nondivergence elliptic equations with VMO coefficients," Transactions of the American Mathematical Society, vol. 336, no. 2, pp. 841-853, 1993.

[9] R. R. Coifman, "A real variable characterization of $H^{p}$," Studia Mathematica, vol. 51, pp. 269-274, 1974.

[10] C. Fefferman and E. M. Stein, " $H^{p}$ spaces of several variables," Acta Mathematica, vol. 129, no. 3-4, pp. 137-193, 1972.

[11] L.-E. Persson, M. A. Ragusa, N. Samko, and P. Wall, "Commutators of Hardy operators in vanishing Morrey spaces," in Proceedings of the 9th International Conference on Mathematical Problems in Engineering, Aerospace and Sciences (ICNPAA '12), vol. 1493, pp. 859-866, AIP, Vienna, Austria, July 2012.

[12] M. A. Ragusa, "Local Hölder regularity for solutions of elliptic systems," Duke Mathematical Journal, vol. 113, no. 2, pp. 385-397, 2002.

[13] E. M. Stein, Singular Integrals and Differentiability Properties of Functions, Princeton University Press, Princeton, NJ, USA, 1970. 


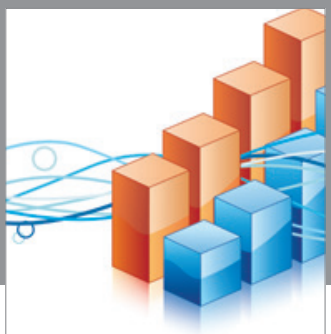

Advances in

Operations Research

vatem alat4

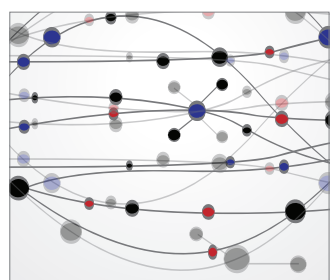

\section{The Scientific} World Journal
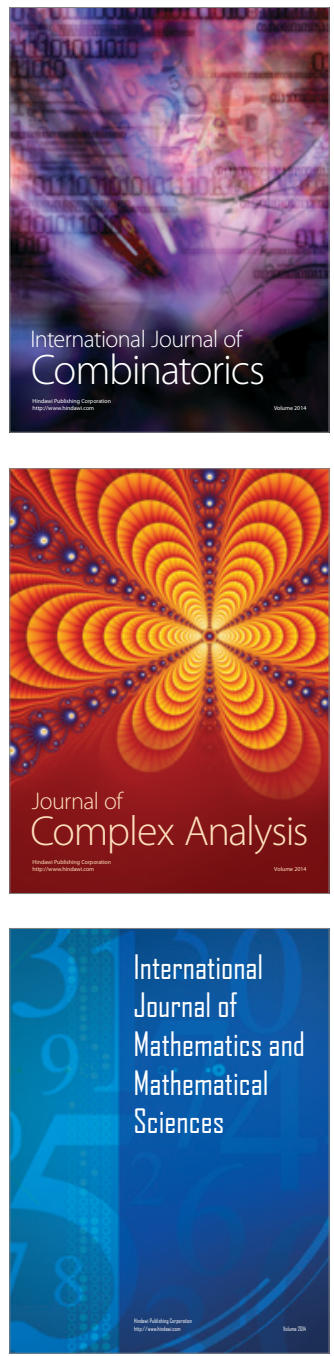
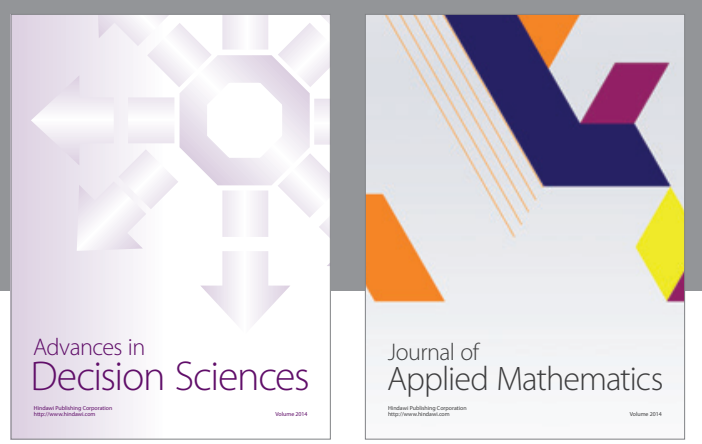

Algebra

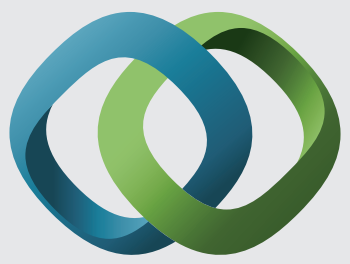

\section{Hindawi}

Submit your manuscripts at

http://www.hindawi.com
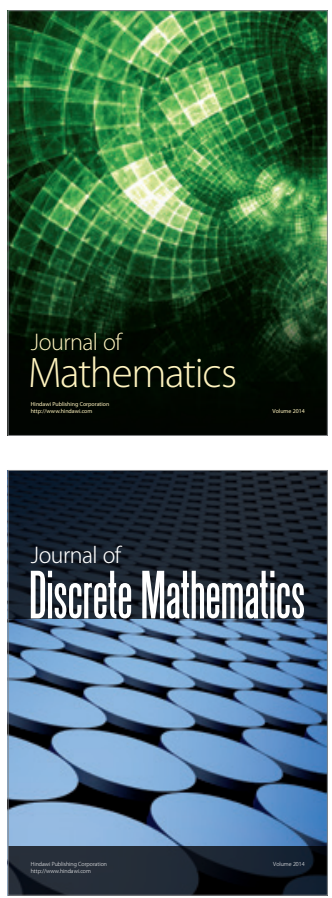

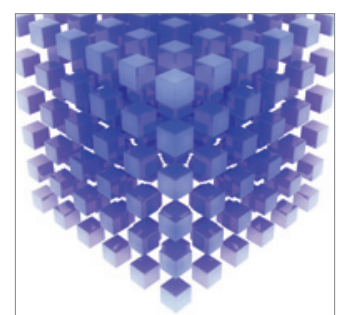

Mathematical Problems in Engineering
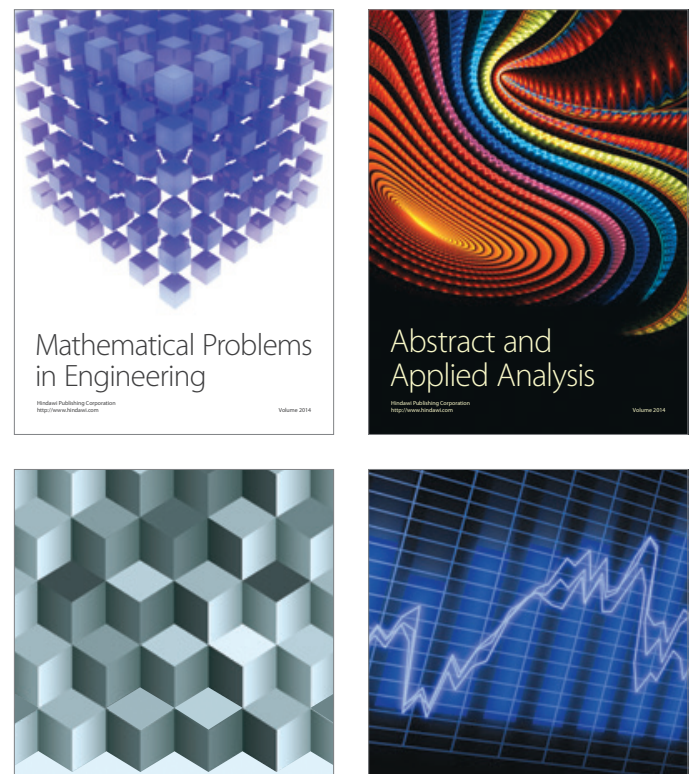

Journal of

Function Spaces

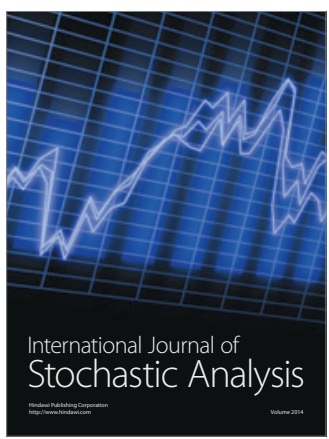

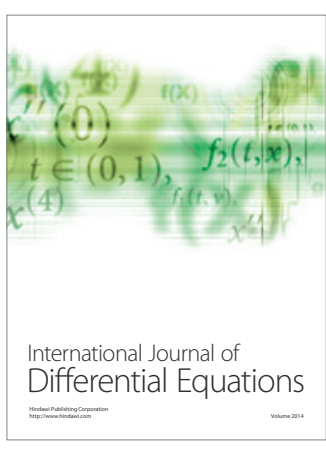
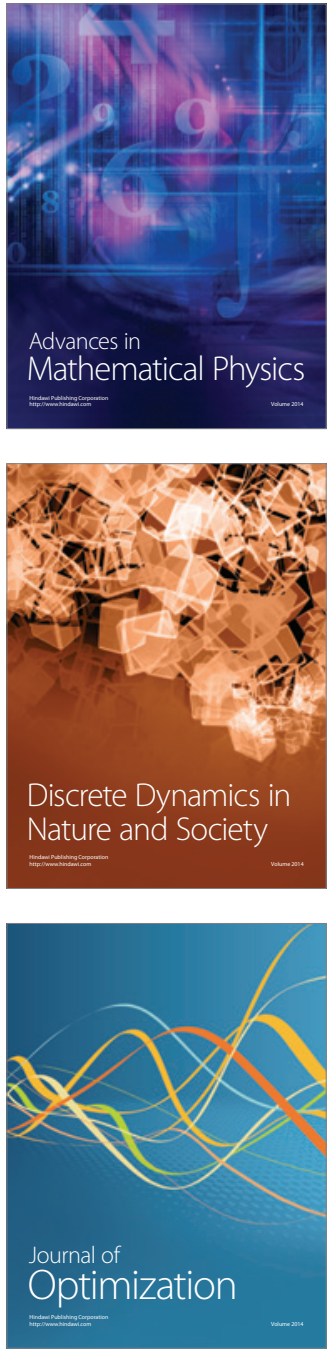